\title{
Prospective constraints on Lorentz violation from ultrahigh-energy photon detection
}

\author{
Grigory Rubtsov, ${ }^{1,2,3}$ Petr Satunin, ${ }^{1}$ and Sergey Sibiryakov ${ }^{1,4,5}$ \\ ${ }^{1}$ Institute for Nuclear Research of the Russian Academy of Sciences, \\ 60th October Anniversary Prospect, 7a, 117312 Moscow, Russia \\ ${ }^{2}$ Faculty of Physics, Moscow State University, Vorobjevy Gory, 119991 Moscow, Russia \\ ${ }^{3}$ Novosibirsk State University, Pirogov street 2, 630090 Novosibirsk, Russia \\ ${ }^{4}$ Physics Department, Theory Group, CERN, CH-1211 Geneva 23, Switzerland \\ ${ }^{5}$ FSB/ITP/LPPC, École Polytechnique Fédérale de Lausanne, CH-1015 Lausanne, Switzerland
}

(Received 16 December 2013; published 26 June 2014)

\begin{abstract}
We point out that violation of Lorentz invariance affects the interaction of high-energy photons with the Earth's atmosphere and magnetic field. In a certain parameter region this interaction becomes suppressed and the photons escape observation, passing through the atmosphere without producing air showers. We argue that a detection of photon-induced air showers with energies above $10^{19} \mathrm{eV}$, implying the absence of suppression as well as the absence of photon decay, will put tight double-sided limits on Lorentz violation in the sector of quantum electrodynamics. These constraints will be by several orders of magnitude stronger than the existing ones and will be robust against any assumptions about the astrophysical origin of the detected photons.
\end{abstract}

DOI: 10.1103/PhysRevD.89.123011

PACS numbers: 96.50.sd, 03.30.+p, 11.30.Cp

\section{INTRODUCTION}

One of the important open questions in astroparticle physics is the presence of a photon component in the ultrahigh-energy cosmic rays (UHECR). An answer to it will help to discriminate between different hypotheses about UHECR production and composition. The recent progress in the UHECR observations has revealed a cutoff in the cosmic ray spectrum at energies $\sim 5 \times 10^{19} \mathrm{eV}$ [1-3]. This is consistent with the prediction made long ago by Greisen, Zatsepin, and Kuzmin (GZK) [4,5] that the flux of primary protons would be depleted at these energies due to pion production on the cosmic microwave background (CMB). Neutral pions subsequently decay into photons. Thus, if the GZK nature of the observed cutoff is confirmed, the fraction of photons in the cosmic rays with energies $10^{19}-10^{20} \mathrm{eV}$ is predicted at the level $10^{-4}-10^{-2}$ depending on the unknown radio background that affects the propagation of photons through the interstellar medium [6]. If the cosmic ray primaries are nuclei instead of photons, the cutoff is the result of nuclei photodisintegration on the cosmic infrared background and CMB [7] and the predicted ultrahigh-energy (UHE) photon flux is two orders of magnitude lower $[6,8]$. UHE photons may also come directly from nearby astrophysical transient sources [9]. They also appear in the "top-down" models of UHECR production, such as superheavy dark matter decay or Z-burst [6]. The photon flux predicted by the latter models is actually higher than the existing bounds, so these models are already disfavored as the primary source of UHECR $[10,11]$. Still, they may be allowed at the level of a subdominant contribution.
No UHE photon detection has been reported so far. However, the upper bounds on the UHE photon flux obtained by the current experiments [10-16] are getting close to the value predicted from the GZK process and one may expect observation of UHE photons in the near future [17].

The physics of cosmic rays is known to be highly sensitive to possible departures from Lorentz invariance (LI) at high energies $[18,19]$. The motivations for such departures, mainly rooted in models of quantum gravity, and their implications for various branches of physics have been extensively discussed in the literature; see the recent review [20] and references therein. The effects of Lorentz violation (LV) are conveniently parametrized within the effective field theory framework [21,22] that assumes existence of a preferred frame, commonly identified with the rest-frame of the CMB. In this frame UHECR particles have a huge energy which may lead to the enhancement of $\mathrm{LV}$ and to observable deviations from the standard physics.

It has been proposed $[23,24]$ that a detection of UHE photon flux compatible with the GZK prediction will impose strong constraints on $\mathrm{LV}$ in the sector of quantum electrodynamics (QED). Assuming that the primary cosmic rays are extragalactic protons and that all UHE photons come from the GZK process, these papers simulate the propagation of UHE photons through the interstellar medium and their resulting flux on Earth in a family of LV models. They showed that, depending on the region in the LV parameter space, the flux will be either highly suppressed due to the vacuum decay of photons into $e^{+} e^{-}$; or, on the contrary, it will be enhanced above the existing bounds due to the weakening of the UHE photon depletion 
through pair production on the CMB. Similar arguments were used earlier in [25] to set the constraints on LV in QED from observations of $\mathrm{TeV}$ gamma rays.

In this paper we point out that not only the photon propagation but also its interactions with the Earth's atmosphere and magnetic field are sensitive to LV. This effect must be taken into account in the analysis of the experimental signatures of LV. Because of it the detection of several photons with energies $10^{19}-10^{20} \mathrm{eV}$ compatible with the standard signatures will lead to very restrictive constraints on LV parameters - at least an order of magnitude stronger than in $[23,24]$. Importantly, these constraints will be robust against any assumptions about the origin and propagation of UHE photons towards the Earth. The idea to use interactions with the atmosphere for constraints on LV was first proposed in [26] in the case of hadronic UHECR primaries. It was discussed in the context of $\mathrm{TeV}$ gamma rays in [27].

\section{INTERACTION OF PHOTONS WITH THE ATMOSPHERE AND LORENTZ VIOLATION}

In the standard LI picture, a primary UHE photon reaching the Earth interacts in the atmosphere and produces an extensive air shower of particles with lower energies that can be detected by the ground-based experiments. The characteristics of the shower are sensitive to the altitude, at which the first interaction initiating the shower occurs. This, in its turn, is determined by the cross section of the first interaction. At energies $\sim 10^{19} \mathrm{eV}$ the dominant channel of the first interaction is $e^{+} e^{-}$pair production on nuclei in the atmosphere-the Bethe-Heitler (BH) process. At higher energy $\left(10^{20} \mathrm{eV}\right.$ and above) the pair production in the geomagnetic field becomes important, leading to the formation of a preshower above the atmosphere. The photon showers initiated by these processes can be identified by the cosmic ray detectors using various observables [28]. As we now discuss, the cross section of the first interaction, and consequently the characteristics of the shower, are strongly affected by LV.

Consider the BH process. The standard result for the cross section reads

$$
\sigma_{\mathrm{BH}}=\frac{28 Z^{2} \alpha^{3}}{9 m^{2}}\left(\log \frac{183}{Z^{1 / 3}}-\frac{1}{42}\right),
$$

where $m$ is the electron mass, $\alpha$ is the fine structure constant, and $Z$ is the nucleus charge; for scattering on nitrogen $(Z=7)$ this gives $\sigma_{\mathrm{BH}} \approx 0.51 \mathrm{~b}$. One observes that, up to factors of order one, the formula (1) follows from a simple dimensional analysis. The mass $m$ in the denominator appears because it characterizes the momentum transfer in the process, while the numerator is obtained by multiplying the coupling constants at the vertices of the corresponding Feynmann diagrams. Depending on the photon energy and the density at the point of the first interaction, destructive interference between several scattering centers somewhat suppresses the $\mathrm{BH}$ cross section - the Landau-Pomeranchuk-Migdal (LPM) effect. The maximal suppression occurs for the final configuration with equal energies of electron and positron and is about a factor of two [28].

The generic effect of LV is the modification of the dispersion relations of photons and electrons/positrons. At momenta smaller than the scale of LV, which is commonly identified with the Planck mass $M=10^{19} \mathrm{GeV}$, these can be expanded in the powers of momenta

$$
E_{\gamma}^{2}=k^{2}+\sum_{n \geq 3} \frac{a_{n} k^{n}}{M^{n-2}}, \quad E_{e^{\mp}}^{2}=m^{2}+p^{2}+\sum_{n \geq 2} \frac{b_{n}^{\mp} p^{n}}{M^{n-2}} .
$$

Note that we normalize the low-energy velocity of photons to 1 , so the quadratic correction to the photon dispersion relation is absent. To get insight about the role of the additional terms, one notices that they can be considered as effective momentum-dependent masses of the particles

$$
m_{\gamma, \mathrm{eff}}^{2}(k)=\sum_{n \geq 3} \frac{a_{n} k^{n}}{M^{n-2}}, \quad m_{e^{\mp}, \mathrm{eff}}^{2}(p)=m^{2}+\sum_{n \geq 2} \frac{b_{n}^{\mp} p^{n}}{M^{n-2}} .
$$

The presence of these masses changes the kinematics of various reactions. In particular, for $m_{\gamma, \text { eff }}$ larger than the sum of effective masses of the electron and positron the photon decay in a vacuum becomes kinematically allowed. In this case UHE photons decay almost instantaneously into $e^{+} e^{-}$pairs and do not reach the Earth $[19,25]$. We focus on the opposite situation, $m_{e^{\mp}, \text { eff }} \gtrsim m_{\gamma, \text { eff }}$. It is straightforward to see that the characteristic momentum transfer in the $\mathrm{BH}$ process is now given by the overall scale of the effective masses of particles, which we denote as $m_{\text {eff }}$, evaluated at the momentum of the incoming photon. The dimensional analysis then yields an estimate of the cross section,

$$
\sigma_{\mathrm{BH}}^{\mathrm{LV}} \sim \frac{Z^{2} \alpha^{3}}{m_{\mathrm{eff}}^{2}(k)}
$$

This is consistent with the estimate of Ref. [27] obtained for the special case of cubic photon dispersion relation using the concept of the radiation formation length and is confirmed by explicit calculation in a model of LV QED [29]. From (4) we see that, if the effective mass evaluated at the energy of the primary photon significantly exceeds $m$, the $\mathrm{BH}$ process will be strongly suppressed. Nonobservation of such suppression will lead to constraints on $m_{\text {eff }}^{2}(k)$ at $k \sim 10^{19} \mathrm{GeV}$ that will translate into the bounds on the coefficients in the modified dispersion relations. 
Similar reasoning carries over to the case of the photon decay in the Earth's magnetic field responsible for the generation of the preshower, with the difference that the sensitivity to the effective mass in this case is exponential [30].

\section{PROSPECTIVE CONSTRAINTS}

While the above arguments are very general and apply to a wide class of LV extensions of QED, to make the quantitative predictions we focus on a specific model studied in [29]. The Lagrangian in the preferred frame reads

$$
\begin{aligned}
\mathcal{L}= & \bar{\psi}\left(i \gamma^{\mu} D_{\mu}-m\right) \psi-\frac{1}{4} F_{\mu \nu} F^{\mu \nu}+i \varkappa \bar{\psi} \gamma^{i} D_{i} \psi \\
& +\frac{i g}{M^{2}} D_{j} \bar{\psi} \gamma^{i} D_{i} D_{j} \psi+\frac{\xi}{4 M^{2}} F_{k j} \partial_{i}^{2} F^{k j},
\end{aligned}
$$

where $\varkappa, g$, and $\xi$ are dimensionless parameters, and the covariant derivative $D_{\mu}$ is defined in the standard way, $D_{\mu} \psi=\left(\partial_{\mu}+i e A_{\mu}\right) \psi$. Greek indices run from 0 to 3 and are raised and lowered with the Minkowski metric, while the latin indices take the values $1,2,3$ and stand for the spatial components; summation over repeated indices is understood. This Lagrangian contains LV operators of dimensions up to 6 that are rotationally invariant in the preferred frame, gauge invariant, and $C P T$ and parity even. The motivation for restricting to these terms is discussed in detail in [29]. From (5) one obtains the dispersion relations for photons and electrons/positrons of the form (2) with $a_{4}=\xi, \quad b_{2}^{\mp}=2 \varkappa, \quad b_{4}^{\mp}=2 g$, and all other coefficients vanishing. We do not consider the cubic modifications of dispersion relations as they have already been strongly constrained by other types of observations [31]; from the theoretical perspective, they are forbidden by postulating the $C P T$ invariance.

Cross sections of several astrophysically relevant processes following from the Lagrangian (5) have been computed [32] in Ref. [29]. For processes with an $e^{+} e^{-}$pair in the final state the result is expressed in terms of the combination

$$
\omega_{\mathrm{LV}}(x)=-\varkappa k-\frac{g k^{3}}{4 M^{2}}\left(1+3 x^{2}\right)+\frac{\xi k^{3}}{2 M^{2}} .
$$

Here, $x \in[-1,1]$ is defined via the ratio of the momenta of the produced electron and positron projected on the direction of the incoming photon, $\left(p_{e^{-}} \cdot p_{\gamma}\right) /\left(p_{e^{+}} \cdot p_{\gamma}\right)=$ $(1+x) /(1-x)$. A straightforward analysis shows that, if $\omega_{\mathrm{LV}}(x)$ is larger than $\frac{2 m^{2}}{k\left(1-x^{2}\right)}$ for some $x$, vacuum photon decay becomes kinematically allowed. Below we concentrate on the values of $\varkappa, g$, $\xi$ when this does not happen. For negative $\omega_{\mathrm{LV}}(x)<-m^{2} / k$, LV significantly suppresses the cross section of the BH process. In the case of $1 \ll$ $k\left|\omega_{\mathrm{LV}}(1)\right| / m^{2} \ll \alpha^{-4} Z^{-4 / 3}$ the expression for the cross section takes the form [29]

$$
\sigma_{\mathrm{BH}}^{\mathrm{LV}} \simeq \frac{8 Z^{2} \alpha^{3}}{3 k\left|\omega_{\mathrm{LV}}(1)\right|} \log \frac{1}{\alpha Z^{1 / 3}} \cdot \log \frac{k\left|\omega_{\mathrm{LV}}(1)\right|}{m^{2}},
$$

which is smaller than (1) by a factor $m^{2} / k\left|\omega_{\mathrm{LV}}(1)\right|$ (up to the logarithm). Note that this is consistent with the estimate (4) upon identifying $k\left|\omega_{\mathrm{LV}}(1)\right|$ as the precise expression for $m_{\text {eff }}^{2}(k)$. Unlike the standard case, the cross section is peaked at the maximal asymmetry between the momenta of the pair, $x= \pm 1$, hence the appearance of $\omega_{\mathrm{LV}}$ at $x=1$.

A future UHE photon detection by cosmic ray experiments would imply that, on the one hand, the photon decay is kinematically forbidden, and, on the other hand, the cross section of the first interaction is not too much suppressed compared to the standard expectation: otherwise, the photon would go through the atmosphere without developing a shower [33]. Conservatively, we require that the cross section does not differ by more that an order of magnitude [34]. This gives the bounds

$$
\omega_{\mathrm{LV}}(x) \lesssim \frac{2 m^{2}}{k\left(1-x^{2}\right)}, \quad-\frac{10 m^{2}}{k} \lesssim \omega_{\mathrm{LV}}(1) .
$$

Applying these constraints to each term in (6) separately, we conclude that a prospective detection of photons with energies $k \sim 10^{19} \mathrm{eV}$ will constrain the $\mathrm{LV}$ parameters at the level [35]

$$
|\varkappa| \lesssim 10^{-25} ; \quad|g| \lesssim 10^{-7}, \quad|\xi| \lesssim 10^{-7} .
$$

Note that smaller than one constraints on $g, \xi$ imply transPlanckian suppression of the quartic terms in the particle dispersion relations.

The constraints (9) can be compared with the existing bounds. The best laboratory constraint on the parameter $x$ is $|\varkappa|<4 \times 10^{-15}$ [36], which is by ten orders of magnitude weaker than (9). The quartic terms in the photon and electron dispersion relations are constrained, respectively, from the timing of distant gamma sources [37-39] and the analysis of the synchrotron radiation from the Crab Nebula [40] at the level $|\xi|<10^{16},|g|<10^{5}$. Finally, our bounds on the parameters $g, \xi$ are an order of magnitude stronger than those discussed in [23,24]. It is worth stressing that the prospective constraints (9) rely only on the known physics of the Earth's atmosphere and are insensitive to any assumptions about the origin of the UHE photons [41].

\section{HOW MANY PHOTONS ARE NEEDED?}

Let us be more precise and estimate the minimal number of UHE photon detections required to obtain the bounds (9). We focus on a primary photon with energy $10^{19} \mathrm{eV}$ and for simplicity neglect the LPM effect. Let $X_{0}$ be the depth of the first interaction of the photon in the atmosphere. This is a random variable with the exponential distribution $d P / d X_{0}=\left\langle X_{0}\right\rangle^{-1} \exp \left(-X_{0} /\left\langle X_{0}\right\rangle\right)$. The mean value of this 
distribution is determined by the cross section of the first interaction, $\left\langle X_{0}\right\rangle=m / \sigma$, where $m$ is the average mass of the atoms of the air (typically, nitrogen). It is $\left\langle X_{0}\right\rangle \approx$ $50 \mathrm{~g} \mathrm{~cm}^{-2}$ for the standard $\mathrm{BH}$ cross section and increases in the LV case.

The depth of the first interaction for a given shower is not directly observed. What is measured instead is the depth $X_{\max }$ where the number of charged particles in the cascade reaches its maximum [28]. This is shifted with respect to $X_{0}$ by the length of the shower development, $X_{\max }=X_{0}+\Delta X$, where $\Delta X$ is also a random variable, whose statistics can be assumed Gaussian due to the large number of interactions that leads to the development of the cascade. The mean value of $X_{\max }$ for photon showers in the LI theory is $\left\langle X_{\max }\right\rangle \simeq 1000 \mathrm{~g} \mathrm{~cm}^{-2}$ (which roughly coincides with the total vertical depth of the atmosphere) and the fluctuations are $\sim 80 \mathrm{~g} \mathrm{~cm}^{-2}$ [43]. In our analysis we will assume that the mean value and fluctuations of $\Delta X$ do not change in the presence of LV. This is justified, since the secondary interactions in the cascade are less energetic than the first one and the effect of LV on them is weaker [44].

$X_{\max }$ for photon showers can be measured either directly with fluorescence detectors or by surface detectors using the properties of the shower front $[45,46]$. The latter technique has an exposure larger by an order of magnitude, but also larger $X_{\max }$ uncertainty of $50 \mathrm{~g} \mathrm{~cm}^{-2}$ compared to $20 \mathrm{~g} \mathrm{~cm}^{-2}$ for fluorescence detectors. To be conservative, we use the value of $50 \mathrm{~g} \mathrm{~cm}^{-2}$ as an experimental uncertainty for $X_{\max }$.

We simulate $X_{\max }$ for a set of $N$ photon events using the standard LI distribution. Each set is compared to the distribution of $X_{\max }$ in the $\mathrm{LV}$ model using the Kolmogorov-Smirnov test and the values of $\left\langle X_{\max }\right\rangle$ excluded at $95 \%$ and $99 \%$ confidence levels are found. We repeat this procedure multiple times and identify $\left\langle X_{\max }\right\rangle$ excluded at the corresponding confidence levels by more than half of the simulated sets (i.e., we require the statistical power of our predictions to be higher than 0.5). The upper limits on $\left\langle X_{\max }\right\rangle$ obtained in this way for various values of $N$ are shown in Table I. The corresponding upper bounds on the ratio of cross sections of the first interaction in the standard and LV cases are also shown.

TABLE I. Predicted upper bounds on $\left\langle X_{\max }\right\rangle$ and the suppression of the first interaction for $N$ UHE events identified as photons with energy $10^{19} \mathrm{eV}$.

\begin{tabular}{lcccc}
\hline \hline & \multicolumn{2}{c}{$95 \%$ C.L. } & \multicolumn{2}{c}{$99 \%$ C.L. } \\
$N$ & $\left\langle X_{\max }\right\rangle, \mathrm{g} \mathrm{cm}^{-2}$ & $\sigma_{\mathrm{BH}} / \sigma_{\mathrm{BH}}^{\mathrm{LV}}$ & $\left\langle X_{\max }\right\rangle, \mathrm{g} \mathrm{cm}^{-2}$ & $\sigma_{\mathrm{BH}} / \sigma_{\mathrm{BH}}^{\mathrm{LV}}$ \\
\hline 1 & - & - & - & - \\
2 & 1880 & 18.6 & - & - \\
3 & 1380 & 8.6 & 1970 & 20.4 \\
4 & 1270 & 6.4 & 1600 & 13.0 \\
5 & 1225 & 5.5 & 1490 & 10.8 \\
\hline \hline
\end{tabular}

Note that one photon event does not lead to any constraints. Indeed, no matter how strongly the first interaction is suppressed, the photon has the conditional probability of $10 \%$ to interact in the first $100 \mathrm{~g} \mathrm{~cm}^{-2}$ of the atmosphere, provided that it interacts at all [47]. Similarly, two events cannot provide exclusion at $99 \%$ C.L. as even for a flat distribution there is always a $1 \%$ chance that they both happen accidentally in the first $100 \mathrm{~g} \mathrm{~cm}^{-2}$. Thus, at least three detections are required to set meaningful constraints and the exclusion power rapidly grows with $N$. We see from the table that already $N=5$ is enough to constrain the ratio of cross sections to be less than 10 at 99\% C.L. yielding the bounds (9) on the LV parameters.

\section{CONSTRAINTS FROM PRESHOWER}

Even stronger constraints on LV will be obtained in the case of UHE photon events with energies $\gtrsim 10^{20} \mathrm{eV}$ and the preshower signature. The process of the photon decay in the geomagnetic field that leads to the preshower formation is exponentially suppressed until the photon energy reaches a certain value-the property that effectively turns it into a threshold reaction. As shown in [30], the suppression exponent, and hence the threshold energy, is modified by LV. Namely, in the model (5) the photon decay width has the form

$$
\Gamma \propto \exp \left[-\frac{8 m^{3}}{3 k e H \sin \varphi}\left(1-\frac{k \omega_{\mathrm{LV}}(0)}{2 m^{2}}\right)^{3 / 2}\right],
$$

where $H$ denotes the magnetic field, $\varphi$ is the angle between the photon momentum and the magnetic field, and $\omega_{\mathrm{LV}}(0)$ is given by (6). We see that in the presence of LV with $\omega_{\mathrm{LV}}(0)<0$ the suppression exponent is enhanced compared to the standard case, which shifts the effective threshold energy above $10^{20} \mathrm{eV}$. Requiring that the shift is not too large implies [48] $\left|\omega_{\mathrm{LV}}(0)\right| \lesssim 2 m^{2} / k$. Applying this inequality separately to the terms with different $k$-dependence in (6), we conclude that a detection of UHE photons at $k \sim 10^{20} \mathrm{eV}$ with the preshower signature will yield the constraints [49],

$$
|\varkappa| \lesssim 10^{-28} ; \quad|\xi-g / 2| \lesssim 10^{-12} .
$$

Similarly to the case of $10^{19} \mathrm{eV}$ photons, several detections are required for statistically significant exclusion. Note, however, that one expects the exclusion power of the preshower events to be higher as additional information can be gained from the comparison of the photon decay probability in the geomagnetic field with the probability of the $\mathrm{BH}$ interaction in the atmosphere.

\section{CONCLUDING REMARKS}

Two comments are in order. First, we have focused in this paper on the case of UHE photons with energies 
$10^{19}-10^{20} \mathrm{eV}$ that can originate in the GZK process. However, our discussion applies essentially without changes to photon-induced air showers of lower energies. Of course, the obtained bounds on the LV parameters will be weaker in this case: the bounds on $\varkappa$ and $g, \xi$ are inversely proportional to the second and fourth power of the photon energy, respectively. Still, a detection of $10^{17} \mathrm{eV}$ photons is already able to probe Planck-suppressed LV. Moreover, interesting constraints on LV can be derived from the existing data on $\mathrm{TeV}$ photon-induced showers. We leave this study for future.

Second, our analysis implies that if high-energy LV is present in nature at the level exceeding (9), no standard photon showers with energies above $10^{19} \mathrm{eV}$ will be detected by the cosmic ray experiments. Instead, if the resulting $\left\langle X_{\max }\right\rangle$ significantly exceeds the atmospheric depth, the signatures of UHE photons will resemble those of neutrinos: there will be no preshowers and the probability of the first interaction in the atmosphere will be almost independent of the depth. In particular, similarly to neutrinos, such LV photons would be able to produce deep inclined air showers with a zenith angle close to $90^{\circ}$. However, they can still be discriminated from neutrinos using the Earth-skimming channel [50] where LV photons should give no signal due to the absence of tau-leptons in the photon shower.

\section{ACKNOWLEDGMENTS}

We thank Maxim Libanov, Stefano Liberati, Thomas Sotiriou, and Sergei Troitsky for discussions. This work was supported in part by the Russian Federation Government Grant No. 11.G34.31.0047, the President of Russian Federation Grants No. NS-5590.2012.2 and No. MK-1170.2013, by the RFBR Grants No. 11-0201528, No. 12-02-01203, No. 12-02-91323, No. 13-0201293, No. 13-02-12095, and No. 14-02-31429, and by the Dynasty Foundation.
[1] R. U. Abbasi et al. (HiRes Collaboration), Phys. Rev. Lett. 100, 101101 (2008).

[2] J. Abraham et al. (Pierre Auger Collaboration), Phys. Rev. Lett. 101, 061101 (2008).

[3] T. Abu-Zayyad et al. (Telescope Array Collaboration), Astrophys. J. Lett. 768, L1 (2013).

[4] K. Greisen, Phys. Rev. Lett. 16, 748 (1966).

[5] G. T. Zatsepin and V. A. Kuzmin, JETP Lett. 4, 78 (1966).

[6] G. Gelmini, O. E. Kalashev, and D. V. Semikoz, J. Exp. Theor. Phys. 106, 1061 (2008).

[7] J. L. Puget, F. W. Stecker, and J. H. Bredekamp, Astrophys. J. 205, 638 (1976).

[8] D. Hooper, A. M. Taylor, and S. Sarkar, Astropart. Phys. 34, 340 (2011).

[9] K. Murase, Phys. Rev. Lett. 103, 081102 (2009).

[10] G. I. Rubtsov et al., Phys. Rev. D 73, 063009 (2006).

[11] M. Settimo et al. (Pierre Auger Collaboration), in Proceedings of 32nd ICRC, Beijing, 2011, http://www.ihep.ac.cn/ english/conference/icrc2011/paper/.

[12] M. Risse, P. Homola, R. Engel, D. Góra, D. Heck, J. Pękala, B. Wilczyńska, and H. Wilczyński, Phys. Rev. Lett. 95, 171102 (2005).

[13] A. V. Glushkov, D. S. Gorbunov, I. T. Makarov, M. I. Pravdin, G. I. Rubtsov, I. E. Sleptsov, and S. V. Troitsky, JETP Lett. 85, 131 (2007).

[14] A. V. Glushkov, I. T. Makarov, M. I. Pravdin, I. E. Sleptsov, D. S. Gorbunov, G. I. Rubtsov, and S. V. Troitsky, Phys. Rev. D 82, 041101 (2010).

[15] J. Abraham et al. (Pierre Auger Collaboration), Astropart. Phys. 29, 243 (2008).

[16] T. Abu-Zayyad et al. (Telescope Array Collaboration), Phys. Rev. D 88, 112005 (2013).
[17] J. Alvarez-Muniz et al. (Pierre Auger, Telescope Array, and Yakutsk Collaborations), Eur. Phys. J. Web Conf. 53, 01009 (2013).

[18] S. R. Coleman and S. L. Glashow, Phys. Lett. B 405, 249 (1997).

[19] T. Jacobson, S. Liberati, and D. Mattingly, Phys. Rev. D 67, 124011 (2003); Ann. Phys. (Amsterdam) 321, 150 (2006).

[20] S. Liberati, Classical Quantum Gravity 30, 133001 (2013).

[21] D. Colladay and V. A. Kostelecky, Phys. Rev. D 58, 116002 (1998).

[22] V. A. Kostelecky and N. Russell, Rev. Mod. Phys. 83, 11 (2011).

[23] M. Galaverni and G. Sigl, Phys. Rev. Lett. 100, 021102 (2008); Phys. Rev. D 78, 063003 (2008).

[24] L. Maccione and S. Liberati, J. Cosmol. Astropart. Phys. 08 (2008) 027.

[25] F. W. Stecker and S. L. Glashow, Astropart. Phys. 16, 97 (2001).

[26] E. E. Antonov, L. G. Dedenko, A. A. Kirillov, T. M. Roganova, G. F. Fedorova, and E. Y. Fedunin, Pis'ma Zh. Eksp. Teor. Fiz. 73, 506 (2001) [J. Exp. Theor. Phys. Lett. 73, 446 (2001)].

[27] H. Vankov and T. Stanev, Phys. Lett. B 538, 251 (2002).

[28] M. Risse and P. Homola, Mod. Phys. Lett. A 22, 749 (2007).

[29] G. Rubtsov, P. Satunin, and S. Sibiryakov, Phys. Rev. D 86, 085012 (2012).

[30] P. Satunin, Phys. Rev. D 87, 105015 (2013).

[31] L. Maccione, S. Liberati, A. Celotti, and J. G. Kirk, J. Cosmol. Astropart. Phys. 10 (2007) 013. 
[32] The computation carefully takes into account the modification of the Feynman rules in the model (5) compared to the LI case.

[33] Another process that can be responsible for the first interaction is the direct photonuclear reaction. However, its cross section is only $10 \mathrm{mb}(1 / 50$ of the $\mathrm{BH}$ cross section) for photons with energy $10^{19} \mathrm{eV}$ [28].

[34] The large asymmetry between the momenta of the pair in the LV case implies that the energy of the leading particle almost does not degrade, which further suppresses the development of the shower. One expects that this effect will also enhance the fluctuations in the depth of the shower maximum. Not taking this into account leaves our bounds conservative.

[35] One can be more precise and ask how accidental cancellations between the three terms in (6) will affect the bounds. Note that the degeneracy between the first and the two other terms is lifted by detection of several photons with energies differing by a factor of a few. Thus, the bound on the parameter $\varkappa$ is robust. As for the parameters $g, \xi$, a straightforward analysis of Eqs. (8) reveals a degeneracy along the ray $\xi=2 g, g<0$. Here, the prospective constraints get weaker and become

$$
-10^{-7} \lesssim \xi-2 g \lesssim 10^{-4} \sqrt{-g} .
$$

[36] B. Altschul, Phys. Rev. D 82, 016002 (2010).

[37] F. Aharonian et al. (HESS Collaboration), Phys. Rev. Lett. 101, 170402 (2008).

[38] A. Abramowski et al. (HESS Collaboration), Astropart. Phys. 34, 738 (2011).
[39] V. Vasileiou, A. Jacholkowska, F. Piron, J. Bolmont, C. Couturier, J. Granot, F. W. Stecker, J. Cohen-Tanugi, and F. Longo, Phys. Rev. D 87, 122001 (2013).

[40] S. Liberati, L. Maccione, and T. P. Sotiriou, Phys. Rev. Lett. 109, 151602 (2012).

[41] Cf. Ref. [42], which discusses the robustness of the constraints on LV following from the absence of the photon decay.

[42] F. R. Klinkhamer, Phys. Rev. D 82, 105024 (2010).

[43] J. Abraham et al. (Pierre Auger Collaboration), Astropart. Phys. 27, 155 (2007).

[44] Strictly speaking, $\Delta X$ and its fluctuations can increase in the LV case, but accounting for this effect would make the constraints even stronger.

[45] P. Abreu et al. (Pierre Auger Collaboration), in Proceedings of 32nd ICRC, Beijing, 2011, http://www.ihep.ac.cn/ english/conference/icrc2011/paper/.

[46] H. Tokuno et al. (Telescope Array Collaboration), Nucl. Instrum. Methods Phys. Res., Sect. A 676, 54 (2012).

[47] The absolute probability of the photon interaction with the atmosphere cannot be constrained without an a priori assumption about the UHE photon flux, which we want to avoid.

[48] The bound on the positive values of $\omega_{\mathrm{LV}}$ again follows from the absence of vacuum photon decay.

[49] Note that the direction of degeneracy of the constraints (11) in the $(g, \xi)$ plane is different from that appearing in the case of the $\mathrm{BH}$ process, cf. footnote [35].

[50] J. Abraham et al. (Pierre Auger Collaboration), Phys. Rev. D 79, 102001 (2009). 